\title{
A study of aspirin and clopidogrel in idiopathic pulmonary arterial hypertension
}

\author{
I.M. Robbins*, ${ }^{\star \uparrow}$, S.M. Kawut ${ }^{\#, \uparrow, 9 \uparrow, ~ D . ~ Y u n g ~}{ }^{+}$, M.P. Reilly ${ }^{\S, f}$, W. Lloyd*, \\ G. Cunningham*, J. Loscalzo**, S.E. Kimmel ${ }^{\S, \# \#, ~ B . W . ~ C h r i s t m a n * ~ a n d ~ R . J . ~ B a r s t ~}{ }^{+}$
}

ABSTRACT: Idiopathic pulmonary arterial hypertension (IPAH) is characterised by in situ thrombosis and increased thromboxane (Tx) $A_{2}$ synthesis; however, there are no studies of antiplatelet therapy in IPAH. The aim of the current study was to determine the biochemical effects of aspirin (ASA) and clopidogrel on platelet function and eicosanoid metabolism in patients with IPAH.

A randomised, double-blind, placebo-controlled crossover study of ASA $81 \mathrm{mg}$ once daily and clopidogrel $75 \mathrm{mg}$ once daily was performed. Plasma P-selectin levels and aggregometry were measured after exposure to adenosine diphosphate, arachidonic acid and collagen. Serum levels of $\mathrm{TxB}_{2}$ and urinary metabolites of $\mathrm{TxA}_{2}$ and prostaglandin $\mathrm{I}_{2}$ (Tx-M and PGI-M, respectively) were assessed.

A total of 19 IPAH patients were enrolled, of whom nine were being treated with continuous intravenous epoprostenol. ASA and clopidogrel significantly reduced platelet aggregation to arachidonic acid and adenosine diphosphate, respectively. ASA significantly decreased serum $\mathrm{TxB}_{2}$, urinary Tx-M levels and the Tx-M/PGI-M ratio, whereas clopidogrel had no effect on eicosanoid levels. Neither drug significantly lowered plasma P-selectin levels. Epoprostenol use did not affect the results.

In conclusion, aspirin and clopidogrel inhibited platelet aggregation, and aspirin reduced thromboxane metabolite production without affecting prostaglandin $I_{2}$ metabolite synthesis. Further clinical trials of aspirin in patients with idiopathic pulmonary arterial hypertension should be performed.

KEYWORDS: Aspirin, clinical trial, hypertension, platelets, pulmonary

diopathic pulmonary arterial hypertension (IPAH) is characterised by sustained elevation in pulmonary artery pressure and pulmonary vascular resistance, which ultimately leads to right heart failure and death. The pathological findings include medial hypertrophy, intimal fibrosis and in situ thrombosis in the small muscular pulmonary arteries [1]. Platelet aggregation, a prerequisite for thrombus formation, is increased in patients with IPAH, and the degree of platelet activity is associated with functional class and survival $[2,3]$. Soluble Pselectin levels are increased in IPAH, reflecting platelet activation [4].

IPAH is also characterised by abnormal arachidonic acid (AA) metabolism, resulting in alterations in the synthesis of thromboxane (Tx) $\mathrm{A}_{2}$ and prostaglandin $\mathrm{I}_{2}\left(\mathrm{PGI}_{2}\right)$ [5]. TxA $\mathrm{A}_{2}$ is a potent vasoconstrictor and smooth muscle mitogen and promotes platelet aggregation [6]. Previously, it has been demonstrated that the major urinary metabolite of $\mathrm{TxA}_{2}$ (11-dehydro-TxB $\left.(\mathrm{Tx}-\mathrm{M})\right)$ is elevated in patients with IPAH compared with healthy volunteers [5]. Although platelets are the predominant source of $\mathrm{TxA}_{2}$ in healthy volunteers $[7,8]$, the source in IPAH is unknown. Due to the known adverse effects of $\mathrm{TxA}_{2}$ on the pulmonary vasculature, investigators have previously attempted to treat IPAH with Tx synthase inhibitors, however, side effects and lack of efficacy limited these studies $[9,10]$. The failure of Tx synthase inhibitors in IPAH parallels their ineffectiveness in other cardiovascular diseases characterised by increased $\mathrm{TxA}_{2}$ and platelet aggregation, which are, on the contrary, effectively treated with antiplatelet agents.

It has also been shown that the major urinary metabolite (2,3-dinor-6-keto-prostaglandin $\mathrm{F}_{1 \alpha}$ (PGI-M)) of $\mathrm{PGI}_{2}$, a vasodilator that inhibits smooth muscle proliferation and platelet aggregation, is decreased in patients with IPAH [5]. Administration of synthetic $\mathrm{PGI}_{2}$ analogues has
AFFILIATIONS

*Dept of Medicine, Vanderbilt University Medical Center, Nashville, TN,

Depts of ${ }^{\#}$ Medicine

+Paediatrics, College of Physicians and Surgeons, and

'Epidemiology, Joseph L. Mailman School of Public Health, Columbia University, New York, NY, ${ }^{\S}$ Dept of Medicine,

\#\# Center for Clinical Epidemiology and Biostatistics, and

${ }^{f}$ Center for Experimental

Therapeutics, University of

Pennsylvania School of Medicine,

Philadelphia, PA, and

**Dept of Medicine, Brigham and

Women's Hospital, Boston, MA, USA

"Authors contributed equally to this paper.

CORRESPONDENCE

S.M. Kawut, Division of Pulmonary, Allergy, and Critical Care Medicine, Columbia University College of Physicians and Surgeons, PH 8E, Rm 101, 622 W. 168th Street, New York, NY 10032, USA.

Fax: 12123425382

E-mail: sk2097@columbia.edu

Received:

August 172005

Accepted after revision:

November 092005

\section{SUPPORT STATEMENT}

This study was funded in part by National Institutes of Health (NIH; Bethesda, MD, USA) grant RR00645 to the Irving Center for Clinical Research at Columbia University, $\mathrm{NIH}$ grant RR00095 to the General Clinical Research Center at Vanderbilt University, and the New York Presbyterian Hospital Office of Clinical Trials Pilot Grant. I.M. Robbins is supported by NIH grant RR15534. S.M. Kawut is supported by NIH grant HL67771 and the Florence and Herbert Irving Clinical Research Career Award. Sanofi-Synthelabo Inc. (Bridgewater, $\mathrm{NJ}$, USA) provided clopidogrel for this study.

European Respiratory Journa

Print ISSN 0903-1936

Online ISSN 1399-3003 
proven clinically effective, confirming that eicosanoid imbalance contributes to the risks of morbidity and mortality in IPAH [11-13]. An oral agent with a favourable safety profile that reduces $\mathrm{TxA}_{2}$ production and platelet aggregation without decreasing $\mathrm{PGI}_{2}$ levels could, therefore, provide significant benefit to patients with IPAH.

Aspirin (ASA) inhibits platelet aggregation and inactivates cyclooxygenase (COX), which catalyses the first step of $\mathrm{TxA}_{2}$ and $\mathrm{PGI}_{2}$ formation. This irreversible inhibition of $\mathrm{COX}$ normally eliminates further platelet Tx production (as platelets are unable to generate new COX). Therefore, low-dose ASA reduces Tx-M to $\angle 20 \%$ of baseline values and serum $\mathrm{TxB}_{2}$ to $<5 \%$ of baseline values with minimal effects on systemic $\mathrm{PGI}_{2}$ generation $[7,8]$. Clopidogrel inhibits platelet aggregation by preventing platelet binding of adenosine diphosphate (ADP). While clopidogrel could decrease TxA $\mathrm{A}_{2}$ levels by inhibition of ADP-induced platelet activation and secondary AA release and metabolism, it does not directly affect COX-1 or eicosanoid production. Although platelet inhibition is efficacious in systemic vascular disease [14-16], specific antiplatelet therapy with either ASA or clopidogrel has not been evaluated in IPAH.

The aim of this study was to determine the biochemical effects of treatment with ASA and clopidogrel in patients with IPAH. The current authors hypothesised that both ASA and clopidogrel would inhibit ex vivo platelet aggregation and decrease plasma P-selectin levels. It was also hypothesised that platelets are the primary source of $\mathrm{TxA}_{2}$ in patients with IPAH and that low-dose ASA would, therefore, reduce $\mathrm{TxA}_{2}$ production without affecting $\mathrm{PGI}_{2}$ synthesis.

\section{METHODS AND MATERIALS Study subjects}

Patients were recruited from the Pulmonary Hypertension Centers at Vanderbilt University Medical Center (Nashville, TN, USA) and New York Presbyterian Hospital (New York, NY, USA). Inclusion criteria were: 1) diagnosis of IPAH; 2) $\geqslant 18$ yrs of age; 3) New York Heart Association (NYHA) functional class I, II or III; and 4) clinical stability (i.e. without change in pulmonary arterial hypertension medical regimen within 1 month prior to enrolment). Patients who had any of the following were excluded: 1) other forms of pulmonary arterial hypertension; 2) a contraindication to ASA or clopidogrel; 3) thrombocytopenia (defined as platelet count $\left.\leqslant 75,000 \cdot \mu \mathrm{L}^{-1}\right)$; 4) history of intracranial haemorrhage or chronic thromboembolic disease; 5) renal failure; or 6) inability or unwillingness to avoid nonsteroidal anti-inflammatory, ASA, or warfarin use for the duration of the trial. The study protocol was approved by the local Institutional Review Board of each institution (Vanderbilt University Medical Center, Nashville, TN; Columbia University, New York, NY, USA).

\section{Study design}

This study was a double-blind, placebo-controlled, threeperiod crossover study comparing ASA $81 \mathrm{mg}$ once daily with placebo and clopidogrel $75 \mathrm{mg}$ once daily with placebo. After informed consent, patients were instructed to stop warfarin 2 weeks before the baseline evaluation and to refrain from nonsteroidal medications and cigarette use for the duration of the trial. The research pharmacy assembled numbered packages of three bottles (labelled with period 1, 2 or 3 ) containing placebo, ASA, or clopidogrel in capsules, which were identical in colour and taste. Sequence allocation was balanced with respect to treatment with continuous intravenous epoprostenol and centre. All investigators, subjects and laboratory personnel were blinded to the treatment sequence.

Patients were evaluated at baseline and after a 2-week treatment period with each drug; a 2-week washout period followed each treatment period. Patients were assessed in the early morning $(\sim 08: 00 \mathrm{~h})$ and were fasting overnight. Patients maintained a diary of study medication use and recorded any new medications taken during the study period. At each visit, a pill count was performed and the diary reviewed to assess compliance. Haemodynamic data were collected from the most recent right heart catheterisation before enrolment in the study.

The primary outcomes were as follows: 1) platelet aggregometry, 2) serum $\mathrm{TxB}_{2}$ level, 2) urinary $\mathrm{Tx}-\mathrm{M}$ concentration, 3) urinary PGI-M concentration, 4) Tx-M/PGI-M ratio, and 5) plasma P-selectin level. Secondary outcomes included symptoms and side effects.

\section{Methods}

Ex vivo platelet aggregation was assessed at baseline and after each drug period $(2,6$ and 10 weeks) in response to collagen $\left(4 \mu \mathrm{g} \cdot \mathrm{mL}^{-1}\right), \operatorname{ADP}\left(3.3,5\right.$ and $\left.10 \mu \mathrm{g} \cdot \mathrm{mL}^{-1}\right)$, and AA $(4,6,8$ and 10 $\left.\mu \mathrm{g} \cdot \mathrm{mL}^{-1}\right)$. Aggregation was assessed in platelet-rich plasma with an aggregometer. For each agonist, the percentage of maximal light transmission achieved within $6 \mathrm{~min}$ of the addition of the agonist was recorded. Maximal platelet aggregation after addition of an agonist allows most light to be transmitted (e.g. 80\%), whereas inhibition of platelet aggregation blocks light from being transmitted (e.g. 20\%).

Blood from study participants was collected in tubes containing sodium EDTA and centrifuged at $1,000 \times g$ for $10 \mathrm{~min}$, within 30 min of collection, to separate plasma. The Human PSelectin immunoassay kit (R\&D Systems Inc., Minneapolis, MN, USA) was used with protocols provided by the manufacturer. Serum $\mathrm{TxB}_{2}$ and urinary levels of $\mathrm{Tx}-\mathrm{M}$ and PGI-M were analysed using stable isotope dilution methodology with gas chromatography/mass spectrometry, as previously described [9].

\section{Analysis}

The data are expressed as mean $\pm \mathrm{SD}$, median (interquartile range) or frequency (95\% confidence interval), except where otherwise noted. t-Tests, rank-sum tests and Fisher's exact tests were used, as appropriate. Linear mixed-effects modelling was used to assess differences between ASA, clopidogrel and placebo. The models included fixed effects for drug, period, sequence, centre, epoprostenol use and the drug-epoprostenol interaction. Subject number was included as a random effect. Pairwise comparisons between ASA, clopdiogrel and placebo were performed only if there was a significant difference overall between treatment groups. Raw baseline data and least squares means and standard errors from the fully adjusted models are reported. Log transformation was used for the TxM/PGI-M ratio, which was highly skewed; back-transformed results are reported from the multivariate model. 
With $\alpha=0.05$ and $\beta=0.20$, eight subjects were required to detect a 1 SD difference in end-points. To assess drug effects stratified by continuous intravenous epoprostenol use, the sample size was increased to 16 . The number of subjects was further increased to 20 to allow for a $20 \%$ drop-out rate. The trial was closed after enrolling 19 patients due to prolonged recruitment.

Primary analyses were performed by intention-to-treat including all patients; secondary analyses included completers only. A p-value $<0.05$ was considered significant.

\section{RESULTS}

Nineteen patients were enrolled from April 2002 until December 2003 (table 1). Nine (47\%) patients were treated with intravenous epoprostenol at a median (range) dose of 20 (16-35) $\mathrm{ng} \cdot \mathrm{kg}^{-1} \cdot \mathrm{min}^{-1}$, and nine $(47 \%)$ were treated with oral bosentan. The median time from the most recent right heart catheterisation to study entry was 248 (125-369) days. Patients receiving epoprostenol had higher mean right atrial and pulmonary artery pressures than those who were receiving oral therapy. Baseline Tx-M levels were significantly elevated compared with previously reported controls in the present authors' laboratory [17]. Baseline P-selectin levels were similar to control values published by the manufacturer of the assay.

\begin{tabular}{|c|c|c|c|c|}
\hline Characteristic & All & Epo & No epo & p-value ${ }^{\#}$ \\
\hline Subjects $n$ & 19 & 9 & 10 & \\
\hline Age yrs & $43 \pm 15$ & $45 \pm 14$ & $40 \pm 16$ & 0.52 \\
\hline Female & $14(74)$ & $6(67)$ & $8(80)$ & 0.63 \\
\hline Ethnicity/race & & & & 0.63 \\
\hline Hispanic/Caucasian & $2(11)$ & & $2(20)$ & \\
\hline Non-Hispanic/ & $4(21)$ & $2(22)$ & $2(20)$ & \\
\hline \multicolumn{5}{|l|}{ African-American } \\
\hline Non-Hispanic/Caucasian & $13(68)$ & $7(78)$ & $6(60)$ & \\
\hline NYHA functional class & & & & 0.57 \\
\hline 1 & $3(16)$ & 2 (22) & $1(10)$ & \\
\hline ॥ & $9(47)$ & $3(33)$ & $6(60)$ & \\
\hline III & $7(37)$ & $4(45)$ & $3(30)$ & \\
\hline \multicolumn{5}{|l|}{ Haemodynamics } \\
\hline RAPm mmHg & $5 \pm 4$ & $8 \pm 5$ & $3 \pm 2$ & 0.009 \\
\hline PAPm mmHg & $46 \pm 18$ & $54 \pm 19$ & $39 \pm 15$ & 0.07 \\
\hline $\mathrm{Cl} \mathrm{L} \cdot \mathrm{min}^{-1} \cdot \mathrm{m}^{-2}$ & $2.9 \pm 1.4$ & $2.4 \pm 1.3$ & $3.3 \pm 1.4$ & 0.15 \\
\hline PVR Wood units & $9.8 \pm 7.9$ & $12.6 \pm 9.4$ & $7.2 \pm 5.6$ & 0.14 \\
\hline PCWPm mmHg & $9 \pm 4$ & $10 \pm 5$ & $8 \pm 3$ & 0.22 \\
\hline \multicolumn{5}{|l|}{ Biochemical assays } \\
\hline Serum $\mathrm{TxB}_{2} \mathrm{ng} \cdot \mathrm{mL}^{-1}$ & $64 \pm 49$ & $47 \pm 44$ & $80 \pm 50$ & 0.15 \\
\hline Urine Tx-M ng.mg $\mathrm{Cr}^{-1}$ & $0.40 \pm 0.19$ & $0.45 \pm 0.22$ & $0.35 \pm 0.15$ & 0.27 \\
\hline Urine PGI-M ${ }^{\dagger} \mathrm{ng} \cdot \mathrm{mg} \mathrm{Cr}^{-1}$ & $2.5 \pm 3.4$ & $5.1 \pm 3.4$ & $0.2 \pm 0.3$ & 0.0002 \\
\hline Urine TX-M/PGI-M ratio & $2.4 \pm 2.9$ & $0.15 \pm 0.11$ & $4.5 \pm 4.6$ & 0.0003 \\
\hline Plasma P-selectin $\mathrm{ng} \cdot \mathrm{mL}^{-1+}$ & $36 \pm 7$ & $34 \pm 7$ & $37 \pm 7$ & 0.47 \\
\hline
\end{tabular}

Data are presented as mean \pm SD or $n(\%)$. Epo: chronic IV epoprostenol; NYHA: New York Heart Association; RAPm: mean right atrial pressure; PAPm: mean pulmonary artery pressure; $\mathrm{Cl}$ : cardiac index; PVR: pulmonary vascular resistance; PCWPm: mean pulmonary capillary wedge pressure; $\mathrm{TxB}_{2}$ : thromboxane $\mathrm{B}_{2} ; \mathrm{Tx}-\mathrm{M}$ : 11-dehydro-thromboxane $\mathrm{B}_{2} ; \mathrm{Cr}$ : creatinine; PGI-M: 2,3-dinor-6-keto-prostaglandin $\mathrm{F}_{1 \alpha} .{ }^{\#}$ : Epo versus no epo. ${ }^{\circ}: \mathrm{n}=18,{ }^{+}: \mathrm{n}=17$.
All patients received the study treatments in the assigned order. Compliance with the avoidance of nonsteroidal antiinflammatory drug guidelines was $100 \%$. Four patients dropped out of the trial, three due to worsening symptoms of pulmonary arterial hypertension and one due to a transient ischaemic attack. Patients who dropped out were not significantly different from those who completed the study in terms of demographic, haemodynamic, platelet or eicosanoid variables at baseline (data not shown).

There was significant suppression of ex vivo platelet aggregation with ASA and with clopidogrel. The results from ADP 3.3 and $10 \mu \mathrm{g} \cdot \mathrm{mL}^{-1}, \mathrm{AA} 6$ and $10 \mu \mathrm{g} \cdot \mathrm{mL}^{-1}$, and collagen are shown in figure 1; other agonist doses produced similar results. Clopidogrel reduced ADP-induced platelet aggregation compared with ASA and placebo. Both ASA and clopidogrel reduced AA-induced platelet aggregation; however, ASA had a significantly greater effect than clopidogrel $(p<0.001)$. Only ASA reduced collagen-induced platelet aggregation. Drug effects were similar in patients with or without intravenous epoprostenol therapy (data not shown).

ASA reduced serum $\mathrm{TxB}_{2}$, urine $\mathrm{Tx}-\mathrm{M}$ and the urine Tx-M/ PGI-M ratio compared with clopidogrel and placebo (table 2). Overall, ASA reduced $\mathrm{TxB}_{2}$ by almost $80 \%(\mathrm{p}<0.001)$, although there was significant variation among patients. ASA also lowered urine Tx-M by $78 \%$ compared with baseline values $(\mathrm{p}<0.001$; fig. 2a), but had no effect on urine PGI-M. Clopidogrel had no effect on eicosanoid metabolites. ASA and clopidogrel modestly decreased plasma P-selectin levels, although this did not reach statistical significance, and there was a possible sequence effect $(p<0.01)$. There were no significant differences in ASA or clopidogrel effects between patients receiving epoprostenol and those who were not (fig. 2).

There were several minor protocol deviations in the trial. Three patients missed a total of seven doses of study drug; no patient missed more than two doses in any single period. One patient had a colonic polypectomy during the washout between periods 2 and 3. This patient's washout period was extended by 1 week. One patient did not save her first morning urine sample, and a second, fasting, morning sample was collected for analysis. Five patient assessments for ADP, six patient assessments (for three patients) for AA, and two patient assessments for collagen were missing or technically inadequate. There was one missing PGI-M value.

There were no significant differences between the effects of the study drugs on NYHA functional class or adverse events. Two patients complained of bruising, one while receiving placebo and one while receiving clopidogrel. One patient complained of haemorrhoidal bleeding and one reported nausea while taking clopidogrel. There were no significant period or carryover effects, other than for P-selectin, as noted. When only patients who completed the entire study were analysed (15 out of the 19 who enrolled), results were consistent with the intention-to-treat analysis (data not shown).

\section{DISCUSSION}

This is the first randomised, double-blind, placebo-controlled clinical trial to evaluate the biochemical effects of antiplatelet 

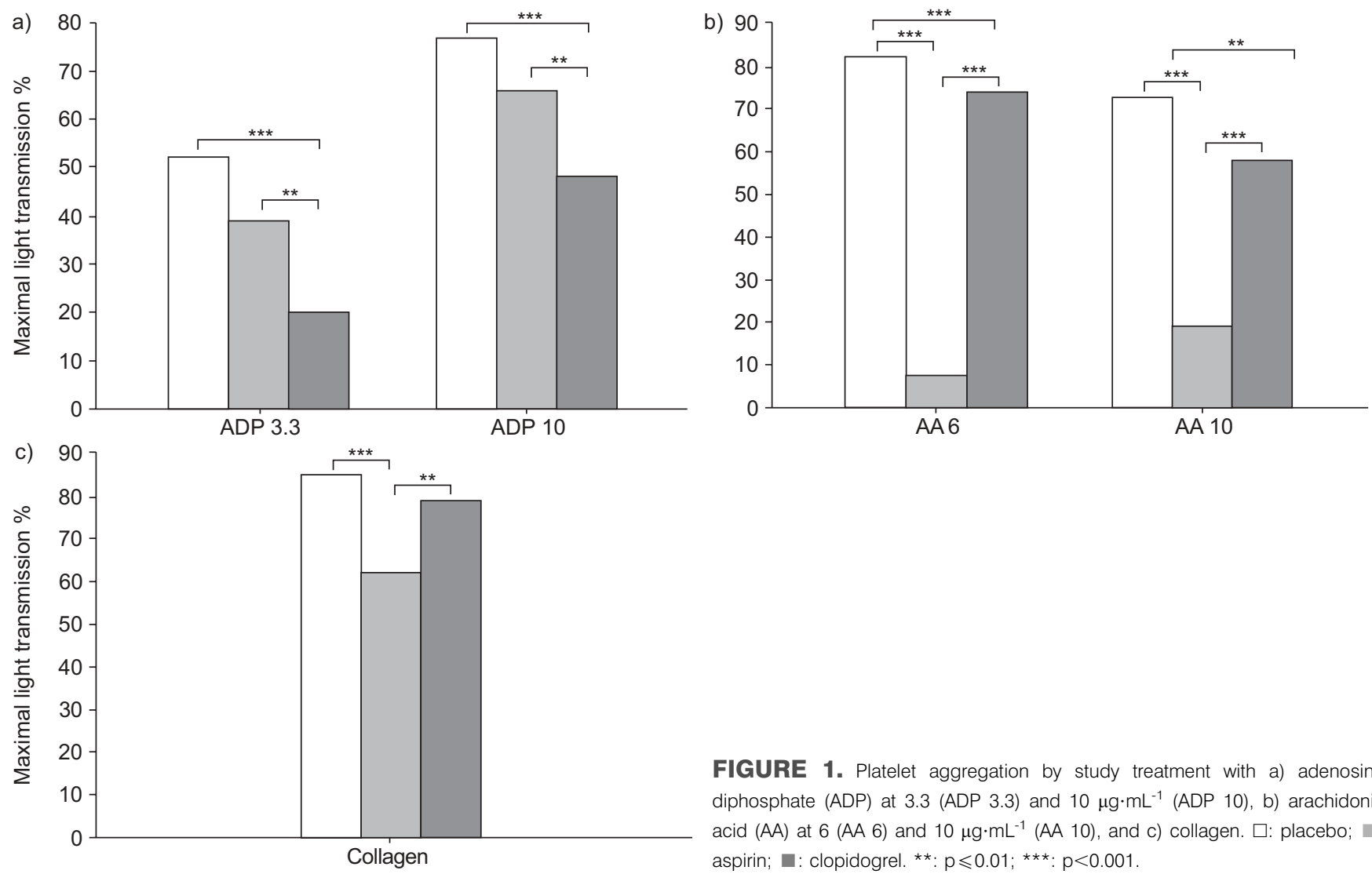

FIGURE 1. Platelet aggregation by study treatment with a) adenosine diphosphate (ADP) at 3.3 (ADP 3.3) and $10 \mu \mathrm{g} \cdot \mathrm{mL}^{-1}$ (ADP 10), b) arachidonic acid (AA) at 6 (AA 6) and $10 \mu \mathrm{g} \cdot \mathrm{mL}^{-1}$ (AA 10), and c) collagen. $\square$ : placebo; aspirin; $\mathbf{a}:$ clopidogrel. ${ }^{* *}: p \leqslant 0.01 ;{ }^{* * *}: p<0.001$

therapy in patients with IPAH. Both low-dose ASA and clopidogrel reduced ex vivo platelet aggregation. ASA also decreased serum $\mathrm{TxB}_{2}$ levels and urine $\mathrm{Tx}-\mathrm{M}$, but not urine PGI-M, therefore significantly improving the Tx-M/PGI-M ratio. Neither ASA nor clopidogrel significantly decreased Pselectin levels, which were normal at baseline.

ASA almost completely inhibited AA-induced platelet aggregation whereas clopidogrel had less of an effect. Conversely, clopidogrel had a much greater effect on ADP-induced aggregation than did ASA. Collagen-induced platelet aggregation, a major stimulus for in vivo aggregation, was inhibited with ASA only. While the extent to which ex vivo studies reflect in vivo conditions remains uncertain, these data confirm a normal inhibitory response of platelets to these agents in patients with IPAH.

ASA was consistent in reducing serum and urine Tx metabolites in all patients in the study. ASA reduced the urinary excretion of Tx-M by almost $80 \%$ of the value on placebo, similar to the reduction seen in both healthy volunteers and in patients with coronary artery disease [7, 8]. Platelets are the major source of $\mathrm{TxA}_{2}$ in these groups, whereas the contributions of vascular, megakaryocytic and other sources to $\mathrm{TxA}_{2}$ production in IPAH are currently unknown. While similar decreases were seen in serum $\mathrm{TxB}_{2}$ levels, previous studies of healthy volunteers reported $94-100 \%$ suppression of serum $\mathrm{TxB}_{2}$ with ASA [8, 18-20]. As serum

\section{TABLE 2 Eicosanoid metabolites and plasma P-selectin after drug administration}

\begin{tabular}{|c|c|c|c|c|c|c|}
\hline \multirow[t]{2}{*}{ Variable } & \multirow[t]{2}{*}{ Placebo } & \multirow[t]{2}{*}{ ASA } & \multirow[t]{2}{*}{ Clopidogrel } & \multicolumn{3}{|c|}{$\mathrm{p}$-value } \\
\hline & & & & $\begin{array}{l}\text { ASA versus } \\
\text { placebo }\end{array}$ & $\begin{array}{c}\text { Clopidogrel versus } \\
\text { placebo }\end{array}$ & $\begin{array}{l}\text { ASA versus } \\
\text { clopidogrel }\end{array}$ \\
\hline Serum $\mathrm{TxB}_{2} \mathrm{ng} \cdot \mathrm{mL}^{-1}$ & $63 \pm 9$ & $13 \pm 9$ & $59 \pm 9$ & $<0.0001$ & 0.75 & 0.0003 \\
\hline Urine Tx-M ng $\cdot \mathrm{mg} \mathrm{Cr}^{-1}$ & $0.33 \pm 1.1$ & $0.09 \pm 1.1$ & $0.32 \pm 1.1$ & $<0.0001$ & 0.44 & $<0.0001$ \\
\hline Plasma P-selectin $\mathrm{ng} \cdot \mathrm{mL}^{-1}$ & $35 \pm 2$ & $31 \pm 2$ & $31 \pm 2$ & 0.05 & 0.09 & 0.85 \\
\hline
\end{tabular}

Data are presented as least squares mean $\pm \mathrm{SE}$ after adjustment for period, sequence, centre, epoprostenol use and the drug by epoprostenol interaction. ASA: aspirin; $\mathrm{TxB}_{2}$ : thromboxane $\mathrm{B}_{2}$; Tx-M: 11-dehydro-thromboxane $\mathrm{B}_{2}$; Cr: creatinine; PGI-M: 2,3-dinor-6-keto-prostaglandin $\mathrm{F}_{1 \alpha}$. 

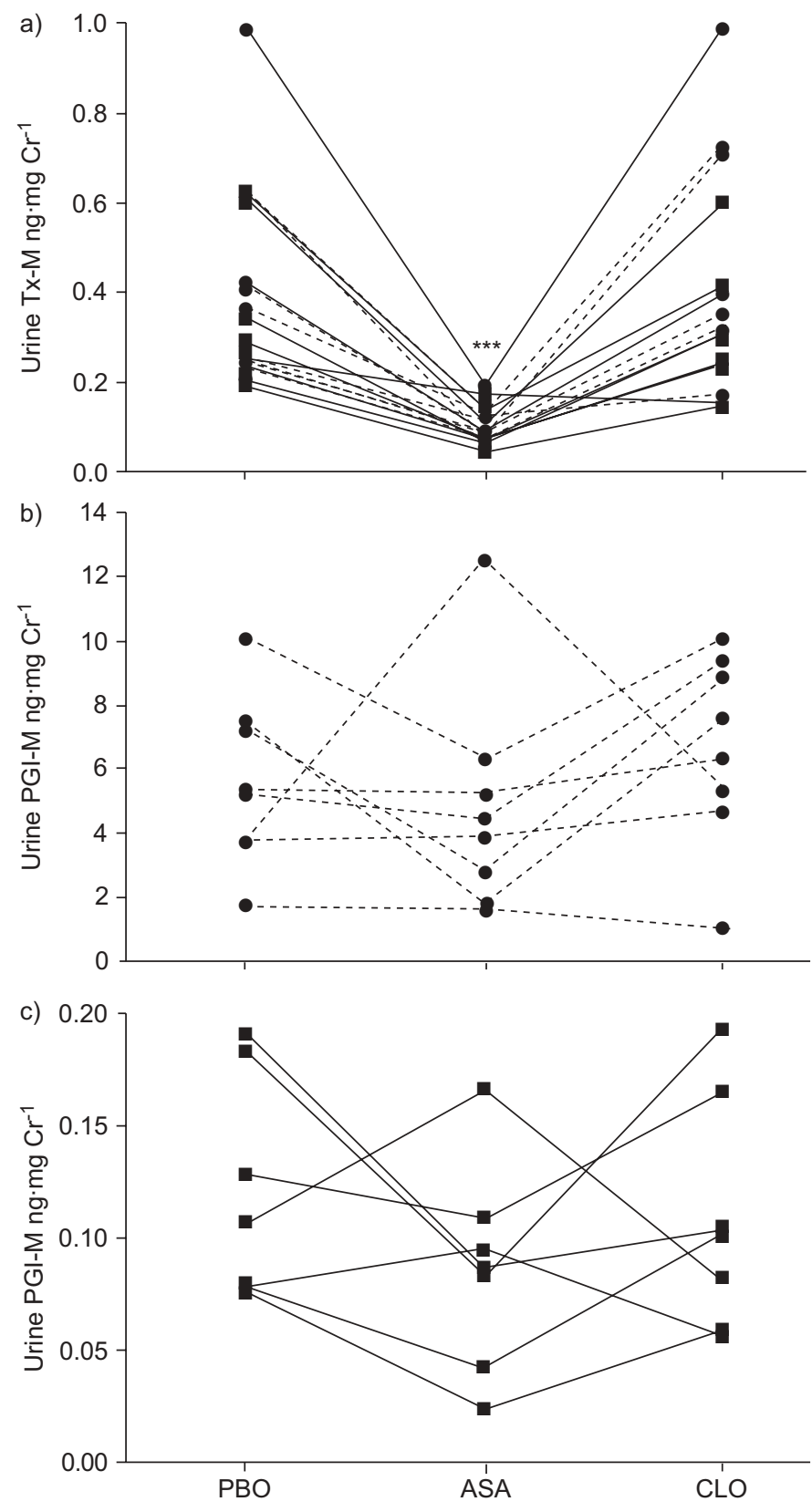

FIGURE 2. a) Urine 11-dehydro-thromboxane $B_{2}$ metabolite (Tx-M), and b) and c) urine 2,3-dinor-6-keto-prostaglandin $\mathrm{F}_{1 \alpha}$ (PGI-M) after drug administration stratified by treatment with epoprostenol $(\bullet)$ or without epoprostenol $(\mathbf{\square})$ for subjects with complete data. Cr: creatinine. ${ }^{* *}: p \leqslant 0.001$ for aspirin (ASA) versus placebo (PBO) and for ASA versus clopidogrel (CLO). All other comparisons were nonsignificant.

$\mathrm{TxB}_{2}$ reflects maximal platelet production, platelet ASA resistance seems the most likely explanation. Alternatively, other cellular sources could account for these findings.

Biochemical ASA resistance is a phenomenon of persistent platelet activation despite ASA administration, measured by in vivo $\left(\mathrm{TxB}_{2}\right)$ or ex vivo (platelet aggregometry) testing. Smaller (but still clinically and statistically significant) reductions were found in $\mathrm{TxB}_{2}$ than were observed with ASA in other patient populations and in normal subjects; however, there was a preserved effect on platelet aggregometry. Insufficient ASA dosing does not explain this finding, as similar doses of ASA (60-80 mg) result in almost complete $\mathrm{TxB}_{2}$ suppression in other reports $[8,19,21]$, and $81 \mathrm{mg}$ is within the recommended dosing range for the majority of cardiovascular indications.

The present authors' previous studies of abnormal eicosanoid imbalance in IPAH suggest an alternative explanation [17]. Nucleated cellular sources of $\mathrm{TxA}_{2}$, such as endothelial cells, macrophages and monocytes, can regenerate COX after ASA administration and possibly account for the residual $\mathrm{TxB}_{2}$ production. This would explain the incomplete $\mathrm{TxB}_{2}$ suppression in the face of robust ASA-induced platelet antagonism, as demonstrated ex vivo. Other potential causes of ASA resistance include increased formation of isoprostanes (nonenzymatic products of lipid peroxidation reflective of oxidant stress) and elevated norepinephrine levels [22-24]. Augmented production of both has been reported in IPAH [25, 26]. Lastly, the increased variability inherent in $\mathrm{TxB}_{2}$ measurement may account for less suppression compared with Tx-M. Regardless, ASA suppressed the majority of Tx production. As TxA $\mathrm{A}_{2}$ is a potent pulmonary vasoconstrictor and smooth muscle mitogen, long-term suppression of this eicosanoid with ASA could prevent progression of disease in IPAH as it does in other cardiovascular diseases.

Clopidogrel had little effect on Tx-M despite substantial inhibition of ADP-dependent ex vivo aggregation. A recent study in healthy volunteers evaluating the effect of therapeutic doses of clopidogrel on ex vivo $\mathrm{TxA}_{2}$ production showed a significant decrease in $\mathrm{TxA}_{2}$; the relevance of this to in vivo effects is unclear [27]. The failure of clopidogrel to decrease $\mathrm{TxA}_{2}$ metabolites in this study suggests that inhibition of ADP-induced aggregation is insufficient to alter platelet AA metabolism. Furthermore, the lack of impact of clopidogrel on collagen-mediated aggregation is consistent with differential effects of antiplatelet therapies on platelet function.

Treatment with continuous intravenous epoprostenol was not randomly assigned and it is, therefore, difficult to draw conclusions about the effect of this therapy on platelet function or other biomarkers in IPAH from these data. Epoprostenol therapy was determined by clinical indication and the availability of other therapies. Not surprisingly, patients receiving epoprostenol tended to be sicker, potentially biasing comparisons between patients receiving epoprostenol and patients who were not. However, certain conclusions may be drawn. Tx-M levels were elevated despite treatment with epoprostenol, consistent with a recent study in which the present authors found that chronic epoprostenol therapy had no effect on urinary Tx-M in IPAH [26]. The results also indicate that the effects of ASA and clopidogrel are similar, regardless of treatment with or without epoprostenol. Importantly, low-dose ASA had a substantial effect on platelet aggregometry and Tx-M levels compared with both placebo and clopidogrel, irrespective of therapy for IPAH.

Plasma P-selectin levels were not elevated in this study population, in contrast with previous studies of patients with PAH [4, 28]. However, the less severe haemodynamic derangements, previous long-term treatment for IPAH and 
clinical stability of the selected study population preclude comparisons with other cohorts. An apparent sequence effect (one sequence of drugs had a higher mean P-selectin level than the others) also weakens the conclusions that may be drawn from this analysis.

There are a number of limitations of this study. First, the crossover study design requires the assumption of disease stability over the time of the trial. Therefore, unstable and NYHA class IV patients were excluded from the study. Even so, several patients had medication dose adjustments and four dropped out of the study, testifying to the fluctuating nature of IPAH, even in apparently stable patients. However, systematic changes in biomarkers over the time of the study and drug-by-period interactions were not found, making significant bias less likely. Analysis of "completers only" resulted in the same findings as the intent-to-treat approach. Secondly, the number of patients enrolled was small, however, the power calculations were conservative and the estimates robust. Thirdly, other markers of platelet activation, e.g. $\beta$-thromboglobulin or platelet factor 4 , were not evaluated, which may have provided additional information regarding the role of platelets in IPAH. Fourthly, this study did not address the effect of epoprostenol on platelet function, which would require a different study design. Finally, concurrent healthy controls were not studied, so comparisons of the effects of ASA and clopidogrel in IPAH must be made with the results of prior research, which has established the effects of these drugs in other populations.

\section{Conclusions}

Aspirin and clopidogrel inhibited platelet aggregation in patients with idiopathic pulmonary arterial hypertension. However, only aspirin significantly inhibited thromboxane $\mathrm{A}_{2}$ synthesis, although not to the full extent seen in previous studies. These results suggest that most circulating thromboxane $A_{2}$ in patients with idiopathic pulmonary arterial hypertension is produced by platelets, although additional cell types, e.g. macrophages or monocytes, may contribute. The present study has shown that aspirin produces significant antiplatelet effects and restores the normal eicosanoid balance in patients with idiopathic pulmonary arterial hypertension. Future studies should focus on the potential clinical benefit and safety of long-term aspirin therapy in pulmonary arterial hypertension.

\section{ACKNOWLEDGEMENTS}

The authors are indebted to the personnel of the Special Coagulation Laboratory at the New York Presbyterian Hospital, the nursing and laboratory staffs of the Irving Center for Clinical Research at Columbia University and the General Clinical Research Center at Vanderbilt University, and A. Ward Scribner at Boston University. The authors are grateful to N.W. Schluger, who provided data and safety monitoring for this study. The authors appreciate the statistical assistance of Y. Wang, M. Elliott and E. Bagiella.

\section{REFERENCES}

1 Pietra GG, Edwards WD, Kay JM, et al. Histopathology of primary pulmonary hypertension. A qualitative and quantitative study of pulmonary blood vessels from 58 patients in the National Heart, Lung, and Blood Institute, Primary Pulmonary Hypertension Registry. Circulation 1989; 80: 1198-1206.

2 Nakonechnicov S, Gabbasov Z, Chazova I, Popov E, Belenkov Y. Platelet aggregation in patients with primary pulmonary hypertension. Blood Coagul Fibrinolysis 1996; 7: 225-227.

3 Lopes AA, Maeda NY, Almeida A, Jaeger R, Ebaid M, Chamone DF. Circulating platelet aggregates indicative of in vivo platelet activation in pulmonary hypertension. Angiology 1993; 44: 701-706.

4 Sakamaki F, Kyotani S, Nagaya N, et al. Increased plasma P-selectin and decreased thrombomodulin in pulmonary arterial hypertension were improved by continuous prostacyclin therapy. Circulation 2000; 102: 2720-2725.

5 Christman BW, McPherson CD, Newman JH, et al. An imbalance between the excretion of thromboxane and prostacyclin metabolites in pulmonary hypertension. $N$ Engl J Med 1992; 327: 70-75.

6 Dorn GW 2nd. Role of thromboxane A2 in mitogenesis of vascular smooth muscle cells. Agents Actions Suppl 1997; 48: Suppl., 42-62.

7 Knapp HR, Healy C, Lawson J, FitzGerald GA. Effects of lowdose aspirin on endogenous eicosanoid formation in normal and atherosclerotic men. Thromb Res 1988; 50: 377-386.

8 Clarke RJ, Mayo G, Price P, FitzGerald GA. Suppression of thromboxane A2 but not of systemic prostacyclin by controlled-release aspirin. N Engl J Med 1991; 325: 1137-1141.

9 Langleben D, Christman BW, Barst RJ, et al. Effects of the thromboxane synthetase inhibitor and receptor antagonist terbogrel in patients with primary pulmonary hypertension. Am Heart J 2002; 143: E4.

10 Rich S, Hart K, Kieras K, Brundage BH. Thromboxane synthetase inhibition in primary pulmonary hypertension. Chest 1987; 91: 356-360.

11 Barst RJ, Rubin LJ, Long WA, et al. A comparison of continuous intravenous epoprostenol with conventional therapy for primary pulmonary hypertension. $N$ Engl J Med 1996; 334: 296-302.

12 Simonneau G, Barst RJ, Galie N, et al. Continuous subcutaneous infusion of treprostinil, a prostacyclin analogue, in patients with pulmonary arterial hypertension: a double-blind, randomized, placebo-controlled trial. Am J Respir Crit Care Med 2002; 165: 800-804.

13 Olschewski H, Simonneau G, Galie N, et al. Inhaled iloprost for severe pulmonary hypertension. $N$ Engl J Med 2002; 347: 322-329.

14 A randomised, blinded, trial of clopidogrel versus aspirin in patients at risk of ischaemic events (CAPRIE). CAPRIE Steering Committee. Lancet 1996; 348: 1329-1339.

15 Fox KA, Mehta SR, Peters R, et al. Benefits and risks of the combination of clopidogrel and aspirin in patients undergoing surgical revascularization for non-ST-elevation acute coronary syndrome: the Clopidogrel in Unstable angina to prevent Recurrent ischemic Events (CURE) Trial. Circulation 2004; 110: 1202-1208.

16 Jagroop IA, Matsagas MI, Geroulakos G, Mikhailidis DP. The effect of clopidogrel, aspirin and both antiplatelet drugs on platelet function in patients with peripheral arterial disease. Platelets 2004; 15: 117-125. 
17 Robbins IM, Barst RJ, Rubin LJ, et al. Increased levels of prostaglandin $\mathrm{D}(2)$ suggest macrophage activation in patients with primary pulmonary hypertension. Chest 2001; 120: 1639-1644.

18 Feldman M, Jialal I, Devaraj S, Cryer B. Effects of low-dose aspirin on serum C-reactive protein and thromboxane B2 concentrations: a placebo-controlled study using a highly sensitive C-reactive protein assay. J Am Coll Cardiol 2001; 37: 2036-2041.

19 FitzGerald GA, Oates JA, Hawiger J, et al. Endogenous biosynthesis of prostacyclin and thromboxane and platelet function during chronic administration of aspirin in man. $J$ Clin Invest 1983; 71: 676-688.

20 Reilly IA, FitzGerald GA. Inhibition of thromboxane formation in vivo and ex vivo: implications for therapy with platelet inhibitory drugs. Blood 1987; 69: 180-186.

21 Hauth J, Sibai B, Caritis S, et al. Maternal serum thromboxane B2 concentrations do not predict improved outcomes in high-risk pregnancies in a low-dose aspirin trial. The National Institute of Child Health and Human Development Network of Maternal-Fetal Medical Units. Am J Obstet Gynecol 1998; 179: 1193-1199.

22 Cipollone F, Ciabattoni G, Patrignani P, et al. Oxidant stress and aspirin-insensitive thromboxane biosynthesis in severe unstable angina. Circulation 2000; 102: 1007-1013.
23 Hurlen M, Seljeflot I, Arnesen H. Increased platelet aggregability during exercise in patients with previous myocardial infarction. Lack of inhibition by aspirin. Thromb Res 2000; 99: 487-494.

24 Larsson PT, Wallen NH, Hjemdahl P. Norepinephrineinduced human platelet activation in vivo is only partly counteracted by aspirin. Circulation 1994; 89: 1951-1957.

25 Nootens M, Kaufmann E, Rector T, et al. Neurohormonal activation in patients with right ventricular failure from pulmonary hypertension: relation to hemodynamic variables and endothelin levels. J Am Coll Cardiol 1995; 26: 1581-1585.

26 Robbins IM, Morrow JD, Christman BW. Oxidant stress but not thromboxane decreases with epoprostenol therapy. Free Radic Biol Med 2005; 38: 568-574.

27 Arrebola MM, De la Cruz JP, Villalobos MA, Pinacho A, Guerrero A, Sanchez de la Cuesta F. In vitro effects of clopidogrel on the platelet-subendothelium interaction, platelet thromboxane and endothelial prostacyclin production, and nitric oxide synthesis. J Cardiovasc Pharmacol 2004; 43: 74-82.

28 Cella G, Bellotto F, Tona F, et al. Plasma markers of endothelial dysfunction in pulmonary hypertension. Chest 2001; 120: 1226-1230. 